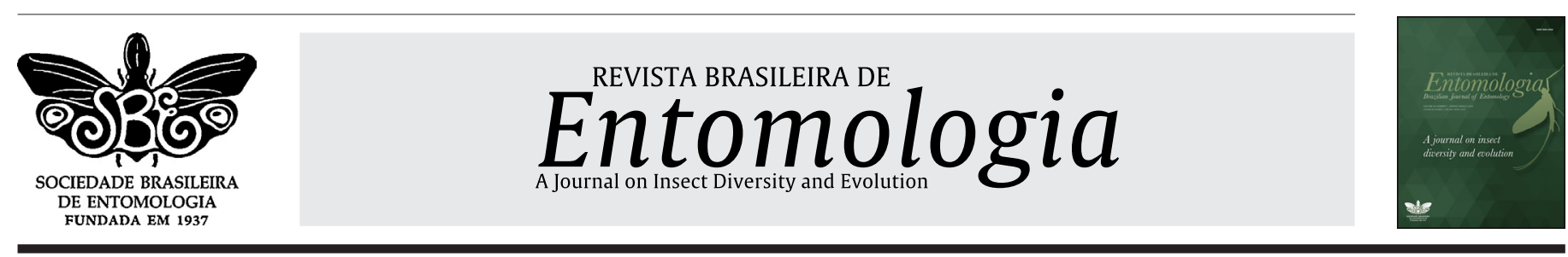

\title{
Taxonomic revision of Dichotomius (Selenocopris) nisus (Olivier, 1719) and Dichotomius (Selenocopris) superbus (Felsche, 1901)
}

\author{
Sheila Cassenote ${ }^{1 *}$ (D), Marcely C. Valois ${ }^{2}$, Maria E. Maldaner ${ }^{3}$, \\ Fernando Z. Vaz-de-Mello ${ }^{3}$ \\ ${ }^{1}$ Universidade Federal de Santa Maria, Centro de Ciências Naturais e Exatas, Santa Maria, RS, Brasil \\ ${ }^{2}$ Universidade Federal do Pará, Instituto de Ciências Biológicas, Belém, PA, Brasil \\ ${ }^{3}$ Universidade Federal de Mato Grosso, Instituto de Biociências, Departamento de Biologia e Zoologia, Cuiabá, MT, Brasil \\ urn:1sid:zoobank.org:pub:9913D2F6-3758-4945-B087-9DA8E1FD7EBC
}

\section{A R T I C L E I N F O}

\section{Article history:}

Received 20 January 2020

Accepted 01 July 2020

Available online 07 September 2020

Associate Editor: Adriana Marvaldi

\section{Keywords:}

Dung beetles

Dichotomiini

Lectotype

New synonyms

\begin{abstract}
A B S T R A C T
Within the subgenus Dichotomius (Selenocopris) Burmeister 1846, D. nisus (Olivier, 1789) and D. superbus (Felsche, 1901) had been historically arranged in the "Nisus" section by Luederwaldt, however, according to the revised classification of the subgenus, the two species now belong to different species groups. In this paper, the taxonomic history concerning the species once included by Luederwaldt in the "Nisus" section is revised and discussed. The following new synonyms are proposed: Pinotus taunayi (Luederwaldt, 1931) as a subjective synonym of D. geminatus (Arrow, 1913), and Pinotus taunayi pilosus (Luederwaldt, 1931) as a subjective synonym of D. nisus. Lectotypes are designated for D. nisus and Pinotus garbei. Redescriptions, diagnoses and updated distribution data are provided for $D$. nisus and $D$. superbus. The diagnostic characters of the male genitalia of these species are described and illustrated for the first time.
\end{abstract}

\section{Introduction}

The genus Dichotomius Hope, 1838 is endemic to the Western Hemisphere and widely distributed in the American continent, with the occurrence of species from the United States to Argentina (Nunes and Vaz-de-Mello, 2013). It currently harbors around 200 valid species, of which about 150 are found in South America (Nunes and Vaz-de-Mello, 2019). Dichotomius is one of the most diverse genera of Neotropical dung beetles. These medium-sized to large beetles occurs mainly in forests, but Dichotomius species can also be found in dry habitats and pastures (Tissiani et al., 2017).

Up to this date, Luederwaldt (1929) provided the most comprehensive revision of the genus under the name Pinotus Erichson (=Dichotomius). In 1951, Martínez suggested a new definition of subgenera proposed by Luederwaldt (1929): D. (Dichotomius) Hope, 1838; D. (Selenocopris) Burmeister, 1846; D. (Homocanthonides) Luederwaldt, 1929 and D. (Luederwaldtinia) Martínez, 1951. After a nomenclatural adjustment proposed by Nunes and Vaz-de-Mello (2019), four subgenera are currently recognized to Dichotomius: D. (Dichotomius) Hope, D. (Cephagonus) Luederwaldt (1929), D. (Selenocopris) Burmeister

\footnotetext{
* Corresponding author:

E-mail: cassenottesheila@gmail.com (S. Cassenote).
}

(1846) and $D$. (Homocanthonides) Luederwaldt (1929). Species belonging to $D$. (Selenocopris) are distinguished from other Dichotomius by the following combination of characters: 1) two clypeal teeth separated by a central emargation; 2 ) rounded head margin with no angulation at the clypeogenal junction (except for some species that exhibit a tooth at this junction); 3) metasternal pilosity, in general, abundant at the lateral lobes and 4) the sixth abdominal ventrite of females not extending beyond the apex of the pygidium nor with a pair of central tubercles (Luederwaldt, 1929; Vaz-de-Mello et al., 2011; Nunes and Vaz-de-Mello, 2019).

The $D$. nisus group was first recognized by Luederwaldt (1929) as the "Nisus" section based on the presence of a single cephalic process on males and an anterior declivity on the pronotum. In addition to D. nisus (Olivier, 1789), this section also included D. superbus (Felsche, 1901), D. lycas (Felsche, 1901) (now in the D. carbonarius group), and $D$. fimbriatus (Harold, 1869) (now in the D. crinicollis group). Luederwaldt (1929) had established 11 sections (i.e. species-group) in $D$. (Selenocopris), with each section containing species which he judged to be more morphologically related. Eighty years later, Gandini and Aguilar (2009) described D. guaranii and considered it as closely 
related to $D$. nisus, but Nunes and Vaz-de-Mello (2019) synonymized D. guaranii with $D$. bicuspis (Germar, 1824).

In 2013, Nunes \& Vaz-de-Mello provided a key to the D. (Selenocopris) species groups, which represents a revised classification of the subgenus. According to this new view of $D$. (Selenocopris), D. superbus is the sole species in its own species group and the same is applied to D. nisus. Therefore, our aim was to provide a taxonomic synopsis of the two poorly known above mentioned species originally assigned to the $D$. nisus species group, despite that our study suggests that the species are not closely related. We do so by providing a detailed literature review, diagnoses, redescriptions, updated distribution data and illustrations of diagnostic characters from both external morphology and male genitalia.

\section{Material and methods}

The study was based on 567 dry-preserved specimens, including the type specimes of all species available names addressed to the "Nisus" section (currently D. nisus species group). The specimens were provided by curators (in parentheses) from the following institutions as listed below:

CEMT - Setor de Entomologia da Coleção Zoológica da Universidade Federal de Mato Grosso, Departamento de Biologia e Zoologia, Cuiabá, Mato Grosso, Brazil (Fernando Vaz-de-Mello)

MNHN - Muséum national d'Histoire naturelle, Paris, France(Olivier Montreuil and Antoine Mantilleri)

MZUFPA - Coleção de Scarabaeinae do Museu de Zoologia, Instituto de Ciências Biológicas, Universidade Federal do Pará, Belém, Brazil (Fernando A. B. Silva)

MZSP - Museu de Zoologia da Universidade de São Paulo, Brazil (Sônia Casari)

SMTD - Staatliches Museum für Tierkunde, Dresden, Alemanha (Olaf Jäger and Klaus-Dieter Klass).

Although we are aware that $D$. nisus is a very common species and other specimens of $D$. superbus are present in other collections, our aim is to present a synopsis, and we do not intent to detail the completeness of their geographical distributions, although no specimens have been examined by us in other collections that came from localities far from the general distributions shown here.

Imaging devices and editing software

Images were obtained with a Leica M205A stereomicroscope, using image stacking software (Leica Application Suite, version 4.2). The images were edited using the Adobe Photoshop CS4 software, and the distribution maps were made using the QGIS version 2.14.2-Essen software.

Taxonomy

Dichotomius (Selenocopris) nisus (Olivier, 1789) (Figs. 1a-g and 3)

Scarabaeus nisus Olivier, 1789: 139, tab.2, fig. 17

Copris nisus: Oliver, 1790: 166; Fabricius, 1801: 44

Copris (Selenocopris) nisus: Burmeister, 1846: not paginated

Pinotus nisus: Harold, 1869: 1010; Gillet, 1911: 61; Luederwaldt: 1914: 369; 1929: 84; Blackwelder, 1944: 207

Pinotus garbei Luederwaldt, 1922: 11; 1929: 84 (synonym of $D$. nisus)

Pinotus taunay ipilosus Luederwaldt, 1931: 303 new synonymy

Dichotomius (Luederwaldtinia) nisus: Marínez, 1951: 140; Vaz-de-Mello, 2000: 193; 2019: not paginated; Nunes \& Vaz-de-Mello, 2013: 418, fig. $2 \mathrm{C}$
Dichotomius nisus: Krajcik, 2006: 54; 2012: 91; Nunes \& Vaz-deMello, 2019: 2239

Type material.

Female Lectotype: Copris nisus Olivier, LECTOTYPE $q$ [here designated, MNHN]: “COLLECTION / OLIVIER / TYPE" // “Pinotus / nisus Oliv" |/ "LECTOTYPE" |/

"Coprisnisus Olivier" / det. F. Vaz-de-Mello".

Male Lectotype: Pinotus garbei Luederwaldt, LECTOTYPE $\precsim$ [here designated, MZSP]: “Cayenne" // "COTIPO" // "Pinotus garbei Lüd. Lüd det. 21" // “17920”. PARALECTOTYPE: $ð$ [MZSP]: “Est. Esp. Santo, Guandú. 2.XI.1920 F. Hoffmann” |/ "COTIPO” // "Pinotus garbei Lüd. Lüd det. 21" // “17921".

Pinotus taunayi var. pilosus Luederwaldt, HOLOTYPE $q$ [MZSP]: "São Paulo. Alto da Serra. R. Spitz leg. v-18" // |/ "COTIPO" |/ "Pinotus taunayi var. pilosus" // “Luederw. Luederw.det.31." // “17846”.

Non-type material

[258 males, 306 females].ARGENTINA: Misiones: Dos de Mayo, XI.2007, E. Abadie-1 2 우 [CEMT]; same data, but XII.67, Juan Foerster-1 $q$ [CEMT]; El Dorado, XII.1994, J.Carlos D’Alessandro-1 $ठ$ 1 [ [CEMT]; Garupa, 24.IV.1995, no collector data-1ð 1 우 [CEMT]; TUCUMAN, Sierra de San Javier, HorcoMolle - yunga, III.2002, Vaz-de-Mello-1 9 [CEMT]. BOLIVIA: Santa Cruz: Botanic Garden

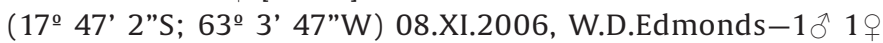
[CEMT]; Chiquitos, 20 km NE Santiago (Tucavaca Valley) (18옥 45.24"S;

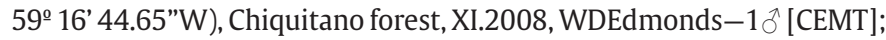
same data, but 1,6Km ENE Santiago (18 12' 3.7”S; 592 21' 0.252”W)2 ㅇ [CEMT]; Florida, Samaipata, pasture (18 10' 46”S; 63 52' 51"W), W.D.Edmonds-1 $\delta$ [CEMT];. BRAZIL: Amazonas: Barreirinha, IX.1991, no collector data-1 + [CEMT].Bahia: Cruz das Almas, I.2005, Ribeiro-Junior g. \& Brito, A.-2우 [CEMT]; Euclides da Cunha, 14.XI.2010, Medina, Campos, Lopes-1우 [CEMT]; Jequié, II.1995, C.Sperber-2 $\lesssim$ đ 1 우 [CEMT]; Milagres, 22.V.2010, MAUlyssêa-2 $q$ [CEMT];same data but, 20.X.2010, Medina, Campos, Ulyssea-1ð [CEMT]; Porto Seguro, RPPN.Veracel. Mussununga, 11.XI.2004, J.N.C.Louzada-2 9 ㅇ [CEMT]; Vila Nova,18.6.1922, Luederwaldt $-3+q 1 \hat{\jmath}$ (MZSP); Ceará: Maranguape, São Benedito Santuário da Nossa Senhora da Penha, Atlantic Forest, (3ํ52' 18”'S; $38^{\circ}$ 40' 53”W), 25.IV.2008, Gillett-1 9 [CEMT].Espírito Santo: Linhares, XI.1989, C. Godinho-1우 [CEMT];samecity, but X.1988, JSSantos-1ð [CEMT]; Venda Nova, IV.1990, E.Grossi-1 ð̄ [CEMT]; Goiás: Bom Jardim de Goiás, II.1997, Vaz-de-Mello-1ð 1 [CEMT]; Mineiros, PNEmas

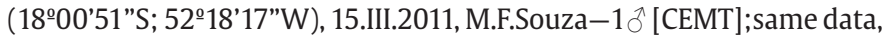

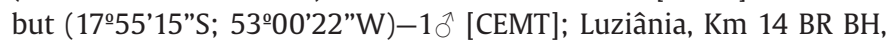
05.XI.1975, Bello-1ð̋ [CEMT].Maranhão: Imperatriz, 21.II.1993, no collector data-1 1 [ [CEMT]; Mirador, Parque Estadual Mirador, Base da Geraldina, X.2006, F.Limeira-de-Oliveira-2우 [CEMT];same data, but Parque Estadual Mirador, Povoado Pindaíba (06²01'06”S; 4500'26”W), VI.2011-1 ð [CEMT]; Santa Quitéria de Maranhão, Faz. Rodiador $47 \mathrm{~m}$

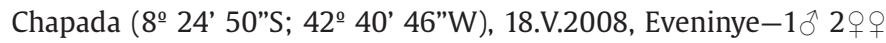
[CEMT].Mato Grosso: Alto Garças, 20.X.2000, R.M.Souza-1 9 [CEMT]; Barão Melgaço, 19.X.1995, M.Gobatto-1ð̂ [CEMT]; Barra do Bugres, Estação Ecológica Serra das Araras, 21.I.1986, S. M. S. Silva-1ठ 1 우 [CEMT]; Chapada dos Guimarães, Cachoeira dos Marimbondos, IV.2010,

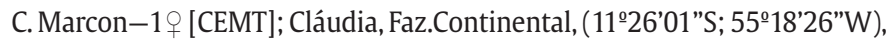
20.II.2011, MFSouza-1ㅇ [CEMT];Consil. 23.IX.2010, P. Barbosa-1ð [CEMT]; Cotriguaçu, Fazenda São Nicolau (949'19”S; 581'18”W),

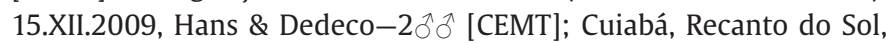
23.IX.2009, C.Dal Mas-1우 [CEMT]; Fazenda Santhidi, 30.XI.2008,

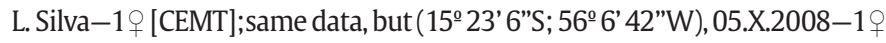
[CEMT];same data, but XI.2011, L.Silva e Oliveira A-1 2 우 [CEMT];same data, but X.2008, L. Silva-1 [CEMT];same data, but IX.2008, R.J. 
Silva-1 12 2 + [CEMT]; same data, but X.2008, LRSilva-3 $3 \hat{\partial}$ [CEMT]; same data, but IX.2008, 1 \& [CEMT]; samelocalitybut XII.1996, Dall'Oglio-1 $\lesssim$ [CEMT];samecitybut 12.XII.1987, PBrondi-1 9 [CEMT];samecitybut 18.IX.1982, AOSilva-1우 [CEMT]; samecitybut 12.X.1988, Pedrina-1우 [CEMT];samecitybut 21.X.1998, PRodrigues-1우 [CEMT];samecitybut 28.X.1994, AMontoura-1 § [CEMT];samelocality, but (15²3'06"S;

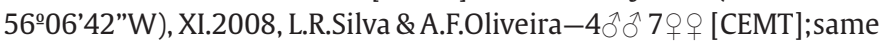
data, but X.2008, L.R.Silva-3 $\widehat{\jmath} 1$ 1 [CEMT]; same data, but IX.20081 우 [CEMT];same data, butlacking date-1우 [CEMT]; Fazenda Niluana, 08.X.1989, MSodrano-1 9 [CEMT]; Diamantino, Vale da Solidão, Cerrado (14²1'52”S; 560ㅜㄹ”W), 26.I.2009, D. Oliveira-1우 [CEMT]; same data, but 19.II.2009-1 J[CEMT]; same data, but 13.I.2001, Génier \& Vaz-de-Mello-1우 [CEMT]; same data, but (142'23”S; 5607'25”W), 19.II.2009-1 $q$ [CEMT]; same data, but 31.I.2009-1 §ै [CEMT]; same

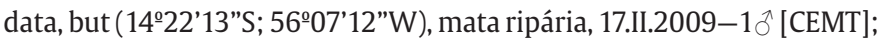

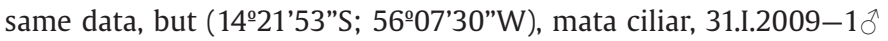
3 우우 [CEMT];samecitybut $5 \mathrm{Km}$ N do Posto Gil on BR 163, Cerrado, 13.I.2001, Génier \& Vaz-de-Mello-1 1 1 [CEMT]; samecitybut Alto Rio

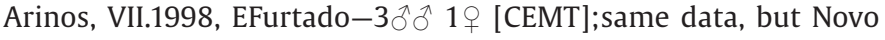
Diamantino, 16.X.2010, MEMaldaner-1 $\partial^{\lambda}$ [CEMT]; same data, but Reserva

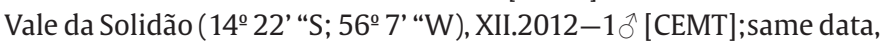

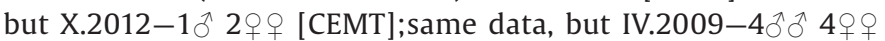

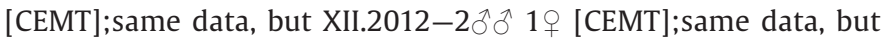
XI.2012-7ð 2 우 [CEMT];same data, but I.2013-3우 [CEMT];same

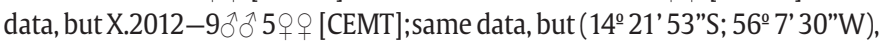
16.II.2009, DCTOliveira-1ㅇ [CEMT]; same data, but (14 22’ 23”s;

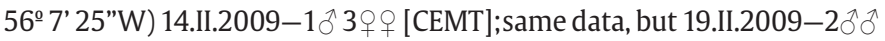
2 우 [CEMT]; Estação Ecológica Buriti, 06.X.1984, Dal Ponte-1ㅇ [CEMT]; samelocality, but (15²4'52"S; 5546'58”W), III.2011, RVNunes-10

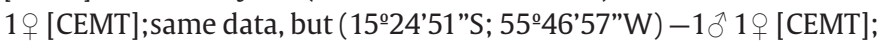

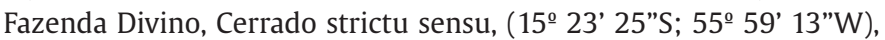
21.II.2013, Daniel Gimo-1 $\odot$ [CEMT]; Fazenda Mutuca, cerrado, 04.X.2008-1 $え$ [CEMT]; same data, but III.2011, RVNunesLGOANunes-1 ${ }^{\lambda}$ [CEMT]; samelocality, but Mata de Galeria, II.2011, RVNunes-1 $\widehat{\partial}$ [CEMT]; Fazenda Sadia, eucalipto, (151' 32”S; 555' 13”W), 21.II.2013, Daniel

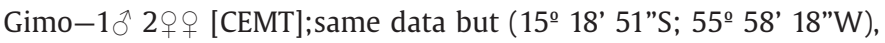
01.II.2013, Daniel Gimo-1 $\delta^{\wedge} 1 q$ [CEMT]; same data, but (15ำ 18' 23"S;

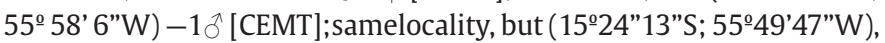
II.2011, RVNunes-1 $\delta^{\lambda} 1$ ㅇ [CEMT]; Fazenda Santhidi, 30.XI.2008,

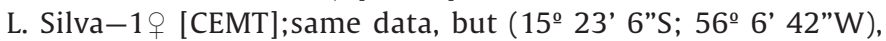
05.X.2008-1 [CEMT]; same data, but XI.2011, L.Silva e Oliveira A-1ð $2 ㅇ ㅜ$ [CEMT];same data, but X.2008, L. Silva-1우 [CEMT];same data, but IX.2008, R.J. Silva-1ð 2 우 [CEMT]; same data, but X.2008, LRSilva-3 ô $^{\wedge}$ [CEMT]; same data, but IX.2008, 1 + [CEMT];samelocalitybut XII.1996, Dall'Oglio-1 § [CEMT]; samecitybut 12.XII.1987, PBrondi-1ㅇ [CEMT];samecitybut 18.IX.1982, AOSilva-1우 [CEMT];samecitybut 12.X.1988, Pedrina-1우 [CEMT]; samecitybut 21.X.1998, PRodrigues-1우 [CEMT];samecitybut 28.X.1994, AMontoura-1 ð [CEMT];samelocality,

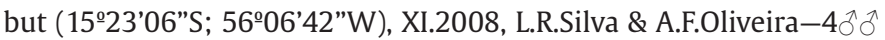

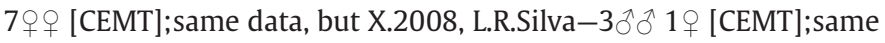
data, but IX.2008-1ㅇ [CEMT];same data, butlacking date-1ㅇ [CEMT]; Fazenda Niluana, 08.X.1989, MSodrano-1ㅇ [CEMT]; Fazenda ToaToa (15²0’30”S; 5551'22”W), II.2011, RVNunes-1ð 1우 [CEMT]; Glória do Oeste, 1.V.2011, ESilva-1 ${ }^{`}$ [CEMT]; Itiquira, Fazenda Araras, (17º 14' 49”S;

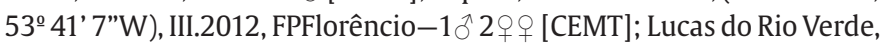
27.XI.2011, BFCamera-1ㅇ [CEMT]; Morro Macumba, (15²0’32"S; 5555'25”W), III.2011, RVNunes-1우 [CEMT]; Nossa Senhora do Livramento, Fazenda Novo Tempo, 25.II.2007, L. Audino - $3 \precsim 2$ 2 ㅇ [CEMT];same data, butPirizal, 15.XI.2012, Simioni-1 9 [CEMT]; Nova

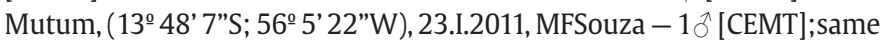
data, but 18.IV.2011-4 $\widehat{\jmath}$ 5우 [CEMT]; Paranatinga, 15.XI.2011, Oneto-1ð [CEMT]; Poconé, 4.XII.1998, J. Moreno T.Mestrec-1ठ

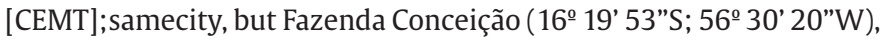

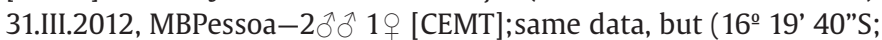

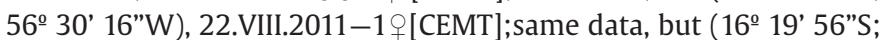

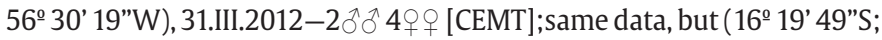

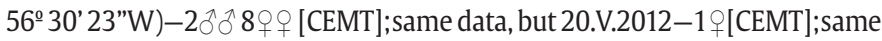

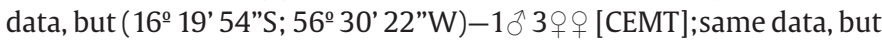

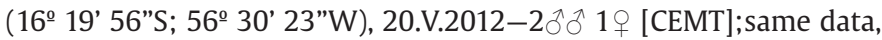

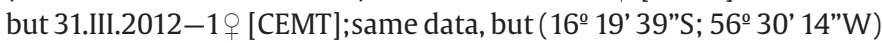

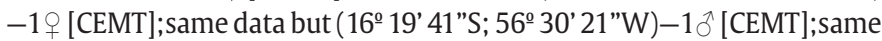

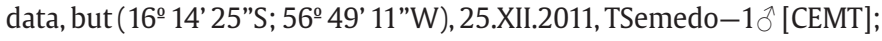
Porto Estrela, Estação Ecologica Serra das Araras, XI.2011, Souza e Gigliotti-1 $\overbrace{}^{\lambda}$ [CEMT]; samelocality, but Final Trilha Boca do José, Parque Cerrado, 15.X.2011, Vaz-de-Mello-5 $\delta^{\curvearrowright} 3$ 우 [CEMT]; samelocality, but Vale do Saloba, Cerrado Sensu Strictu, 14.X.2011-2 ðð [CEMT];same data, but Trilha do Heliporto, Cerrado (15 39' 20"S; 57ํ12' 51"W),

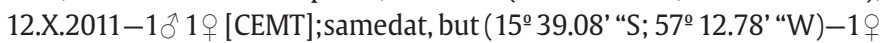

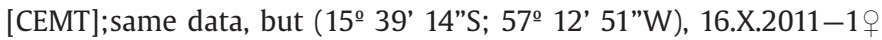

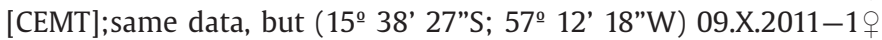
[CEMT]; same data, but Cerrado Rochoso (15 39' 16"S; 57²12' 40"W),

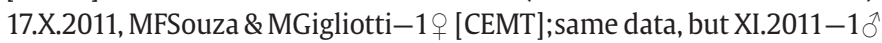

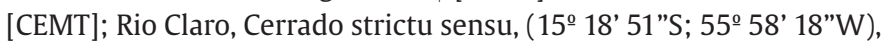
01.II.2013, Daniel Gimo-1우 [CEMT];same data, butgaleryforest, (1520' 9"S; 55ํ5' 0”W), 27.II.2013, Daniel Gimo-2

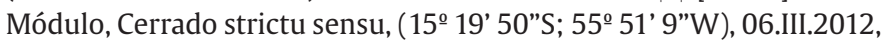
Daniel Gimo-1ㅇ [CEMT]; Santo Antônio de Leverger, 17.XI.2008, V. Kihara $-1 \delta 2$ 우 [CEMT]; samelocality, but 08-XI-2008, I. Lage-1우 [CEMT]; samecity, but Fazenda Santa Maria, 09.III.1992, CRThomas-1 $\widehat{\jmath}$ [CEMT]; samelocality, but 17.II.1992, HRodrigues-1 9 [CEMT]; same data, but LMMoreira-1ㅇ [CEMT]; same city, but São Vicente, 19.X.1988, DMFigueiredo-1우 [CEMT]; same data, butMCCMurta-1우 [CEMT]; samecity, but São Vicente da Serra, 27.VI.2010, AGPereira, -1 우 [CEMT]; Sinop,

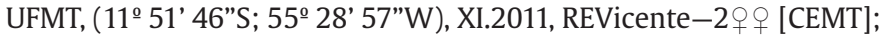

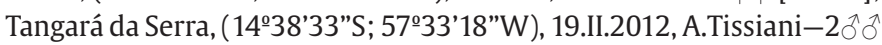
1 우 [CEMT]; samecity, but 2008, RJSilva-1 $\lesssim 1$ 우 [CEMT]; same data, but 2007-19 [CEMT]; UNEMAT [Universidade do Estado do Mato Grosso], no collector data-3 3 đ 3 우 [CEMT]; Várzea Grande, Pirinéu, 29. VIII.1998, SKida-1 9 [CEMT];samecity, but 23.VIII.1993, ETakeda-1우 [CEMT]; samecity, but Parque Boa Vista, samecity, but Parque Boa Vista,

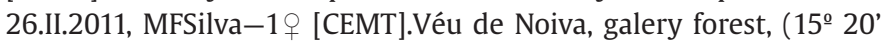
10"S; 55 54' 25”W), 27.II.2013, Daniel Gimo-1 ô 1 ㅇ [CEMT]; same

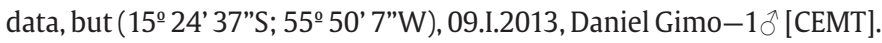
Mato Grosso do Sul: Aquidauana, pastagem de Brachiariasp, X.2011,

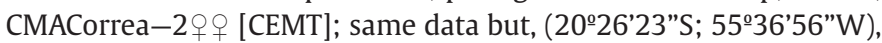

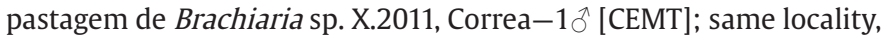
but fragmento de cerrado (20⒉ 26' 54”S; 49 38' 39"W), 15.X.2011, CMACorrea - $1 \delta^{1} 1$ [ [CEMT]; Assentamento Canaã (20 46. 267' "S; 56은 46.038' “W), XI.2009, FORoque-2우 [CEMT]; Bodoquena, Canaã,

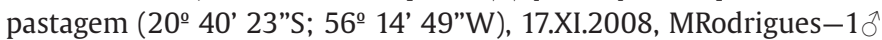
[CEMT]; Bonito, Fazenda Pitangueiras, (20 52' 14"S; 56 35' 19”W)

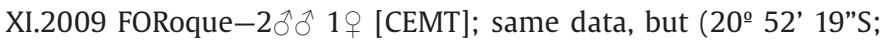

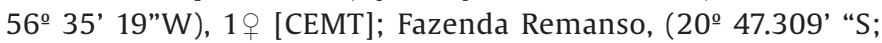
$56^{\circ}$ 49.737' 'W), XI.2009, FORoque-1 [CEMT]; Jardim, Buraco das

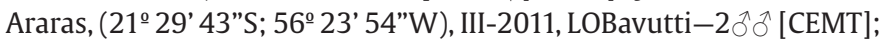
Campo Grande, Cerradão, 24.II.2000, JNC Louzada-1 [ [CEMT]; Corumbá, Passo da Lontra BEP/UFMS, Campo arbustivo, no collector data-1우 [CEMT];samelocality, XI.2000, JRaizer $1 \delta^{\Uparrow} 2$ 우우 [CEMT]; samelocality,

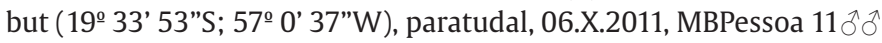
4 우우 [CEMT];same data but, 18.X.1968, Bello-1 ڤิ [CEMT];same data but, Embrapa CNPGC, Brachiariadecubens11.IX.1990, WWKoller-10

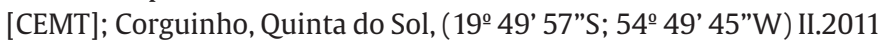
LOBavutti-5 o $^{\lambda} 13$ 우 [CEMT]; Dourados, 12.XI.2005, M. Miloca-1우 [CEMT]; Fazenda Nhumirim, (19 3’ 14”S; 56 40' 26”W). 21.IX.2011, 
MBPessoa; Corguinho-6 3 3우 [CEMT]; Guia Lopes da Laguna, 15. XI.2007, collectorunreadable-1 1 1 [CEMT]; Ivinhema, (22 31' 39"S;

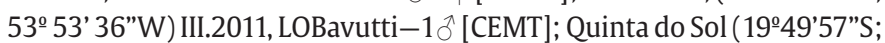
544ㅜ45”W) II.2011, LOBavutti-1 $\lesssim$ [CEMT];Selviria, Unesp Farm, Brachiariadecubens, 19.III.1992, CAHFlechtmann-1우 [CEMT];same data but, 14.III.1996-1 [CEMT]. Três Lagoas, Fazenda Barra do Moeda, XI.2009, P. Rosas-1 $\partial^{\lambda}$ [CEMT].Minas Gerais: Buenópolis, Parque Estadual

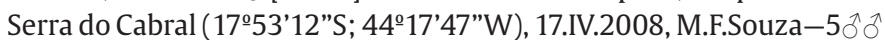

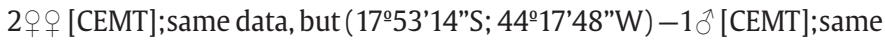

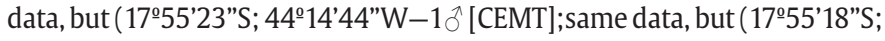

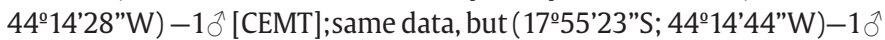

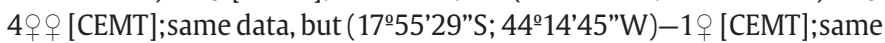

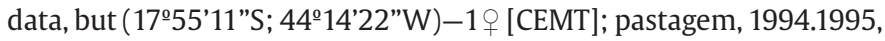
Louzada, Carvalho \& Silva-2 9 [ [CEMT];Caxambú, XII.1990, Bello-1우 [CEMT]; Ipatinga, XI.1991, E. Grossi-1ð 2 $q$ [CEMT]; Cordisburgo, XII.1993, Vaz-de-Mello-1 9 [CEMT];same data, but Fazenda Pontinha, XII.1993,Vaz-de-Mello-3우 [CEMT];same data, but I.1993-1ð 2 우우

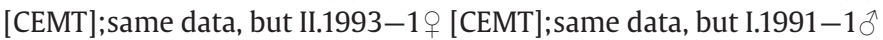
[CEMT];same data, but X.1993-1 + [CEMT];same data, but no collector

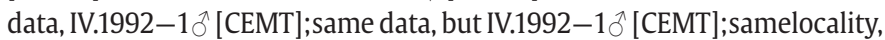
but XI.1983, C. Godinho-2 $\circ$ ㅇ [CEMT]; Diamantina, Campus UFVJM,

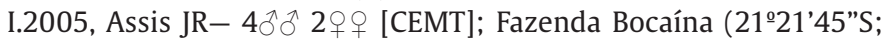
4503'17”W), 22.III.2008, M. R. Santos \& D. L. H. Takahashi-1우

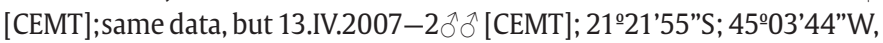
13.IV.2007, Rocha, Sânia, Takahashi-1ㅇ [CEMT];same data, but

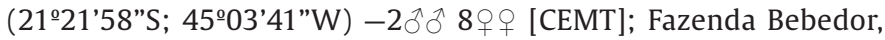
2.IX.1938, Luederwaldt-1 9 (MZSP); Goianópolis, Parque Nacional da

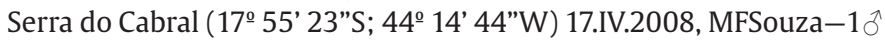
[CEMT]. Lavras, (21ำ18'10.68”S; 44ํ5'22.25”W), 20.I.2008, Rocha M.S.;

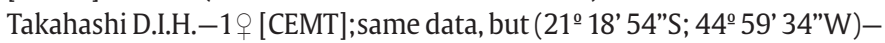

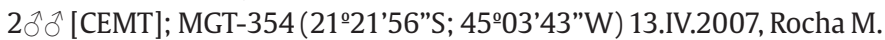
S; Takahashi D. I. H -1 \% [CEMT]; same data, but (2121'55"S;

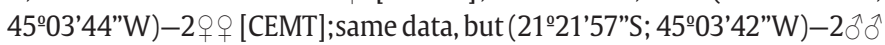

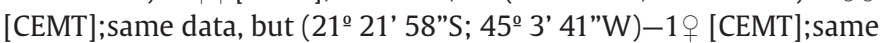

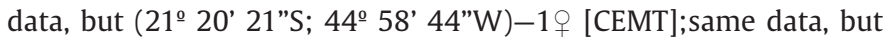

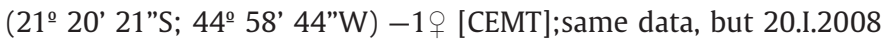

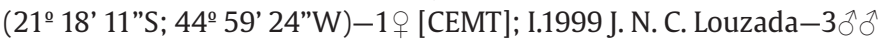
[CEMT]; 27.VI.1998, Marcos A. S. 1 \% [CEMT]; Campus da UFLA, XII.2001, Vaz-de-Mello-1 [CEMT]; Nova Era, III.1987, J. C. Zanuncio-1 $\partial^{\jmath}$ [CEMT]; Parque Nacional da Serra do Cipó, II.2002 J. N. C. Louzada-3우 [CEMT]; Santa Bárbara, Cruzeiro.Peti, afloramento rochoso, 16.X.1998, MFVasconcelos-1우 [CEMT]; Serrinha, 03.III.2007, samecollector-1우 [CEMT]; Três Marias, XI.1991, J. N. C. Louzada-1우 [CEMT];Uberaba, X.1995, Vaz-de-Mello-1 $1_{1}$ [CEMT]; Viçosa, Mata do Paraíso, (2047’S; 4251'W), XII.1998, Vaz-de-Mello-1 §̊ [CEMT]; 23.IX.1993, Louzada, Silva-13 $\widehat{o}^{1} 5$ 우우 [CEMT]; Pará: Alter do Chão, Savana, VII.2003, Matavelli

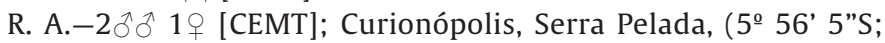
49 40' 17"W), III.2012, MAragona-1 9 [CEMT]. Pau d'Arco, Fazenda

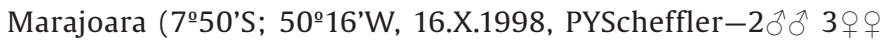
[CEMT]; Paraíba: Mamanguape, Tabuleiro (6ำ1'S; 3510W) II..2002, A.A. Endres -1 \% [CEMT]; Araruna, Parque Estadual da Pedra da Boca

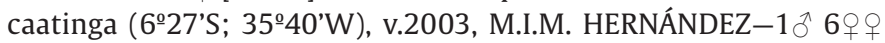
[CEMT]; Paraná: Entre Rios do Oeste, 24.XII.2010, MEMaldaner-1 ${ }^{\lambda}$ [CEMT].Pernambuco: 18.IX.2010, A. Freitas-19 [CEMT]; Floresta, V.2012, RVNunes-1 $ठ$ [CEMT]; Igarassu, 13.I.1999, Kátia-1 $ᄋ$ [CEMT].Piauí:

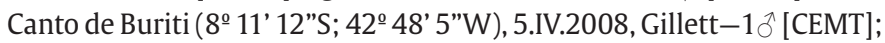
Nonato, Parque Nacional da Serra da Capivara, 16.XII.1998, CARMatragolo-2 + ㅇ [CEMT]; same data, but 29.XI.1998-1 ठ̂ [CEMT]; São Raimundo Nonato, 31.X.1992, no collector data-1 + [CEMT]; Rio de Janeiro: Bagé, 26.X.2011, RMMoraes-1 ${ }^{\wedge}$; same data, but Fazenda Pitangueiras (31ํ41' 4.58"S; 54 37' 25.31"W) 1ㅇ [CEMT]; same data, but 29.II.2012-1ㅇ [CEMT]; Eldorado do Sul, Estação Experimental UFRGS, 5.IV.2012, Moraes \&
Podkowa-15 3 ô 14우 [CEMT];Grajaú, XI.1993, Leonardo-1우 [CEMT]; Itaipava, VII.1991, no collector data-1 $\odot$ [CEMT]. Secretaria Maria Comprida, III.1993, Vaz-de-Mello-2 $\widehat{\partial}$ [CEMT]. RIO GRANDE DO SUL: São Vicente do Sul, XII.1998, DMTakiya-1우 [CEMT]; Três de Maio, XII.2003, CMOliveira-1 đ̂ 1ㅇ [CEMT]; Rondônia: Vilhena, 20.X.2011,

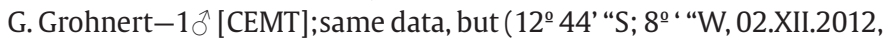
NGrohenert-1 ¿̊ [CEMT].Roraima: Cantá, IX.1997, Ribeiro \& Vaz-de-Mello-1우 [CEMT]; Serra da Moça, lavrado, VII.1997, Vaz-de-Mello-1ðð 1 q [CEMT]. Santa Catarina: Florianópolis, Beira da Praia de Jurerê, 10.XI.2007,

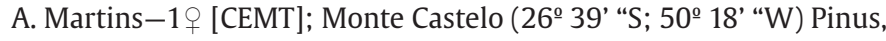
24.XII.2012, ALBrandi-1 $ð$ [CEMT].São Paulo: Descalvado, Fazenda Itaúnas, Mata+Citrus, 09.IV.2008, NWPerioto-1ð [CEMT]; same data, but 22.I.2007-1 [CEMT]; Jundiaí, 30.XII.2011, MMRCastro-1우 [CEMT]; Tupã, rural área, 05.IV.2000, Baggio Filho-1+ [CEMT]; COLOMBIA: Meta: Hacienda La Liberdad, sabana, X.2001, JNoriega-1ð [CEMT]; López, bosque de galeria em sabana, IX.1994, JNoriega-1ð [CEMT];Remolinos, 18.III.1993, G.AlemanJ.Arenas-1 ô [CEMT]; San Martin, HaciendaTocancipa, sabana-bosque, IV.2006, J. Noriega-1우 [CEMT];Villavicencio, IV.1996, Var-1우 [CEMT]; Tolima: Armero, I.1982, E.Gómez. F.Gonzales-1ㅇ [CEMT]; Santander:Cucutilla, Norte de Santander, Sisavita (7º 26' 7"S; 755ㅜㅇ' 6"W), VIII.2001, JNoriega-1우 [CEMT].FRENCH GUYANA: Cayenne: Paracou Field Station ( $\left.5^{\circ} 02^{\prime} \mathrm{N} ; 53^{\circ} 00^{\prime \prime W}\right), 55 \mathrm{~m}$, X. 2003, F. Feer. Leg. $-2 \lesssim ð$ $3 ㅇ$ [CEMT]. PARAGUAY: Boquerón: M Estigarribia, 5-9.V.1992, no collector data-1ठิ [CEMT]; Concepción: Concepción, 20.XI.1995, CAguilar-1 $\delta^{\jmath} 1$ 우 [CEMT]; same locality, but 20.X.2005, Mareti $\left(1{ }^{\lambda}\right)$.

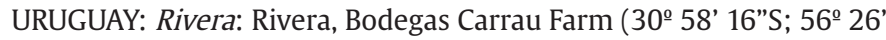
38"W) 11.X.2011, RMMoraes-1 1 1 [CEMT]; same data, but (305ㅗ' 19"S; 56ํㅜㄹ' 40"W)-3 [CEMT].VENEZUELA: Bolívar: Cólon, Hacienda La Honda, IV.1984, JBlanco-1 9 [CEMT]; La Fria, Hacienda La Liberdad, 21.VII.1984, JBlanco-1 $\precsim$ [CEMT]; Rio Pargaza, 100m, 15.V.2004, D. Garcia-1우 [CEMT].

\section{Diagnosis.}

In $D$. (Selenocopris), Dichotomius nisus is similar to D. superbus by having a large body size (length $>14 \mathrm{~mm}$ ), a high conical process on head (Figs. 1a, 2a) and pronotum with a distinct anterior declivity delimited posteriorly by two lobes (Figs. 1a, 2a). However, D. nisus can be separated from $D$. superbus by the following characters: body black; surface of pronotal excavation with transverse ridges; posterior margin of pronotum with a band of ocellate punctures (Figs. 1a, c); pronotal disc with well-impressed median longitudinal sulcus; paramera asymmetric, left paramere with a strong apical hook (Figs. 1d, e); lamella copulatrix (LC) not sclerotized medially, except for a median elongated projection (Fig. 1f).

Redescription ( $\left(^{\top}\right)$. Length: 14-23 mm. Maximum width (pronotum): $17 \mathrm{~mm}$. Body black, except teneral specimens. Copper to yellow setae on the ventral surface. Head. Clypeus distinctly marginate anteriorly. Anteroventral margin with a row of setae, longer along the genal margin, setae visible in dorsal view. Clypeal margin with two rounded teeth separated by a V-shaped emargination (Figs. 1a-c). Anterior margin weakly angulated on the clypeo-genal junction.Genal margin slightly surpassing the clypeal margin. Surface slightly to moderately transversely ridged. Ventral clypeal process bifurcated. Clypeo-frontal region with a median high conical horn, emarginate at the apex (only on large males) (Figs. 1a-c). Antenna with nine antennomeres; antennal club elongated, covered by short and light brown setae. Pronotum. Wider than long. Antero-median portion widely excavated (Figs. 1a, c). Surface of pronotal excavation, lateral portion of the disc and anterior angles with transversal granules (Figs. 1a, c). Disc distinctly convex, surface densely coated with shallow punctures (Figs. 1a, c). Hypomeron. Not excavated anteriorly and lacking transverse carina medially. Median portion almost entirely smooth, except by the presence of scattered 
seitiferous punctures. Anterior portion densely covered by setiferous punctures, except on glabrous area near antero-lateral portion of the circumnotal ridge which bears ocellate punctures spaced by approximately their diameter. Prosternum. Surface with spaced ocellate setigerous punctures; central portion weakly convex. Mesoventrite. Smooth medially, lateral portions densely covered by setiferous ocellate punctures. Meso-metaventral suture regularly arched. Metaventrite. Anterior margin with a shallow V-shaped impression medially. Anterior lobe not angulated. Metasternal disc finely punctate. Anterior lobe with setiferous punctures, separated by three times their diameter, distributed throughout the lateralmargin. Lateral lobe densely coated with punctures bearing long setae. Mesepimeron and metepisternum. Densely coated with punctures bearing long setae. Elytra. Striae thin, punctate, lateral margin carinate. Punctures of striae spaced by twice their diameter, deeper than striae. Surface of interstriae slightly convex, chagrineed, less shiny than pronotum. Legs.external margin of protibiae quadridentate. Apical spur of protibiae acute, curved down on apical third, angulated on external margin. Ventral surface of profemur with
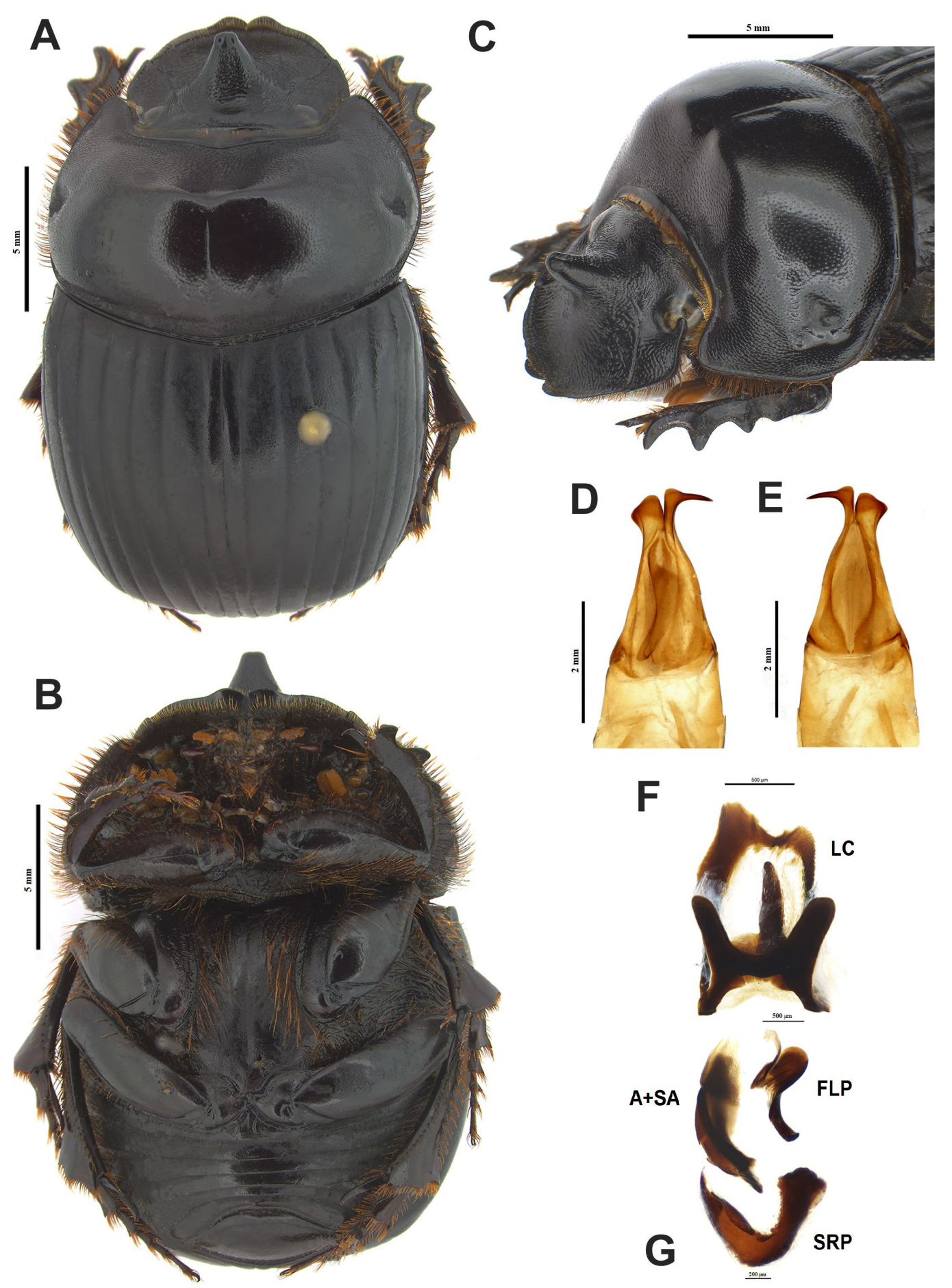

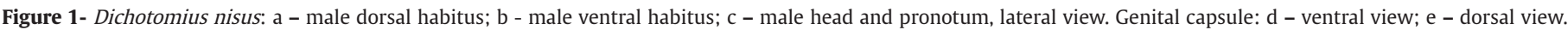

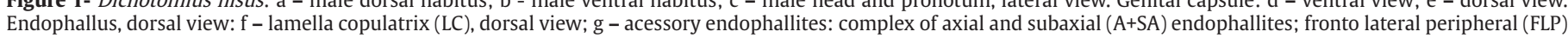
endophallites; superior right peripheral (SRP) endophallites. 
ocellate setiferous punctures along the midline. Ventral surface of meso- and metafemurs with sparse fine punctures. Ventral surface of meso- and metatibiae smooth. Meso and metatibia widely expanded apically. Apical margin of metatibia transverse, not distinctly angulate medially. Apical spur of metatibiae apically truncate. Abdomen. Ventrites narrowed medially. Anterior margin of ventrites with sparse ocellate punctures. Sixth ventrite as long as fifth medially. Pygidium. Slightly convex, wider than long. Disc bearing fine punctures equally spaced. Anterior margin with a row of ocellate punctures. Genital capsule. Paramera sub-triangular, 2/3 as long as phallobase (Figs. 1d, e). Lateral margin of right paramereslight angulate at apical fourth (Fig. 1e). Ventral sclerotized valves of paramera S-shaped (Fig. 1d). Endophallus. Superior right peripheral endophallite (SRP) U-shaped, rigth portion pointed, left portion widened (Fig. 1g). Fronto-lateral peripheral endophallite (FLP) C-shaped with an anterior projection (Fig. 1g). Complex of axial and subaxial ( $A+S A)$ endophallites elongated, comma-shaped, subaxial endophallite with reticulate texture, covering the axial endophallite on superior portion (Fig. 1g). Lamella copulatrix (LC) sub-retangular, not sclerotized medially, inferior margin with three elongated projections (Fig. 1f). Female. It differs from the male by the head cephalic carina very low, with a pair of feeble tubercles. Sixth abdominal ventrite twice the size of the fifth ventrite and slightly curved medially.

Type locality. "Cayenne" [French Guyana].

Distribution. Dry, open habitats in French Guyana, Venezuela, Colombia, Brazil, Bolivia, Argentina, Paraguay and Uruguay.

Taxonomic remarks. Scarabaeus nisus was described by Olivier (1789), and the description is clearly based on a female; a specimen bearing a green circular TYPE label (which indicate that it was a part of the original Olivier collection now at the Olivier collection housed in MNHN). There is no clear indication that Olivier (1789) had only one specimen, we follow the Recommendation 73F of ICZN (1999) and this specimen is here designated as the lectotype of Scarabaeus nisus.

Pinotus garbei (Luederwaldt, 1922) was described based on older specimens (e.g. having the clypeus worn), either way, Luederwaldt (1922) mentioned the similarity between P. garbei and P. nisus twice, including the apical hook on the aedeagus present in both species. Later, P. garbei was synonymized by Luederwaldt (1929) himself.

Luederwaldt (1922) described $D$. taunayi based on several specimens from northwest Brazil. The species was superficially characterized by the male pronotum with anterior declivity and impressed medialy, posterior margin of the pronotum with ocellate punctures and elytral striaedistinct punctate. Within $D$. (Selenocopris) the species of the group D. geminatus (Arrow, 1913) have the anterior portion of pronotum with an median impression. After comparing the syntypes of $D$. taunayi housed in MZSP we conclude that the specimens used for description of described $D$. taunayi actually belongs to the previously described species $D$. geminatus. This species, along with $D$. opacipennis and $D$. mysticus were included in the $D$. geminatus group as defined by Nunes and Vaz-de-Mello (2013). Within the group, D. opacipennis and $D$. geminatus have the pronotum as wide as elytral. However, only $D$. geminatus has the posterior margin of the pronotum with ocellate punctures and elytral striae deeply punctate. While D. opacipennis is distributed throughout out dry habitats of central Brazil, D. geminatus has been recorded in the atlantic forest and costal habitats from the northwest and north of Brazil, therefore, including the known distribution of $D$. taunayi. Thus both morphology and distribution suggest that $D$. taunayi is a junior subjective synonym of $D$. geminatus.

Pinotus taunayi pilosus (Luederwaldt, 1931) was described as a variety of Pinotus taunayi Luederwaldt, 1922. We examined the types of both names and they belong to different species. The type specimen of P. taunayi pilosus have an old red label where can be read "COTIPO" suggesting that it is part of a type series. However, as stated in the original publication, Luederwaldt (1931) based on a single female from Alto da Serra (São Paulo State, Brazil) to describe the variety P. taunay ipilosus. We found a single specimen of $P$. taunay ipilosus in Luederwaldt's collection hosted at the MZSP, but the specimen is a less developed male rather than a female as stated by Luederwaldt (1931). Based on the pronotum puncture, striae and interstriae punctuation and edeago with left paramere having the distinct strong apical hook, there is no doubt that the specimen is, in fact, an minor male of $D$. nisus. In order to maintain nomenclatural stability we have assigned a lectotype to P. taunayi.

We propose that Pinotus taunayi is a new subjective synonym of D. geminatus (Arrow, 1913), while Pinotus taunay ipilosus is a new synonym of $D$. nisus. We believe that Pinotus taunay ipilosus was described as a variety of Pinotus taunayi due to subtle similarities between small specimens of $D$. nisus and D. geminatus (herein considered senior synonym of $D$. taunayi).

Dichotomius (Selenocopris) superbus (Felsche, 1901) (Figs. 2a-g and 3) Pinotus superbus Felsche, 1901: p. 145 (original description); Gillet, 1911: 62; Luederwaldt, 1929: 84; Blackwelder, 1944: 208

Pinotus (Selenocopris) superbus: Luederwaldt, 1929: p. 86-87

Dichotomius (Luederwaldtinia) superbus: Vaz-de-Mello, 2000: 193; 2019: not paginated; Nunes \& Vaz-de-Mello, 2013: 418, fig. 2 E.

Dichotomius superbus: Krajcik, 2006: 56; 2012: 92

Type material. Pinotus superbus Felsche, HOLOTYPE $\curvearrowright$ [SMTD]: "superbus | Felsche | Parâ" || "Pará" || "Typus" || "Coll. C. Felsche” || "Kauf 201918 // HOLOTYPE $\widehat{O}$ ”.

Non-type material. BRAZIL: Rio Grande do Sul, Porto Alegre, [no collectorand date data] $-1+$ [CEMT]. São Paulo, Cantareira, [no collector data], $1940-1 ð$ [CEMT]. ARGENTINA: Misiones: Loreto. IX-57. [no collector data] [ $2 \lesssim \precsim 2+q$ CEMT], same data, but III-60-1 [CEMT].

\section{Diagnosis.}

Among the species currently assigned to D. (Selenocopris), $D$. superbus and D. nisus have a following combination of characters: large body size (length $>14 \mathrm{~mm}$ ), a conical process on head, pronotum with a distinct anterior declivity delimited posteriorly by a bilobate knob (Figs. 1a and 2a). However, only D. superbus have the dorsum metallic green, usually with red reflections on pronotum and elytra. Apex of male cephalic process rounded, not emarginate. Elytral striae I-VII slightly impressed, smooth (Fig. 2a). Lateral margins of pronotum distinctly marginate, margin irregularly serrate throughout (Figs. 2a, c). Surface of anterior excavation of the pronotum covered with dense ocellate punctures (Figs. 2a, c). Pronotal disc densely coated with shallow punctures (Figs. 1a, c). Parameres 1/3 shorter than phallobase (Figs. 2d, e). Apex of paramera truncate, left paramera lacking projection or hook at the externalmargin (Fig. 2d). LC approximately double hook-shaped, median posterior portion with a tongue-like projection with one-fifth the length of LC in dorsal view (Fig. 2f).

Redescription (ð̋). Length: 15-17 mm. Maximum width (pronotum): $10 \mathrm{~mm}$. Body oblong, moderately convex, dorsal surface almost entirely metallic green, usually with red reflections (Figs. 2a-c). Light brown to yellow setae on the ventral surface. Head. Clypeus distinctly marginate anteriorly. Anteroventral margin with a row of setae, longer on the genal margin; setae visible in dorsal view. Clypeal margin distinctly bidentate and slightly upturned medially, teeth rounded separated by a deep U-shaped emargination (Figs. 2a, b). Surface densely punctate, shallowly to moderately impressed punctures, except for smooth area localized anteriorly to the cephalic process. Clypeofrontal region with a high conical horn medially, distally truncates (Figs. 2a, c). The surface of front slightly impressed medially. Clypeal ventral process pronounced into 

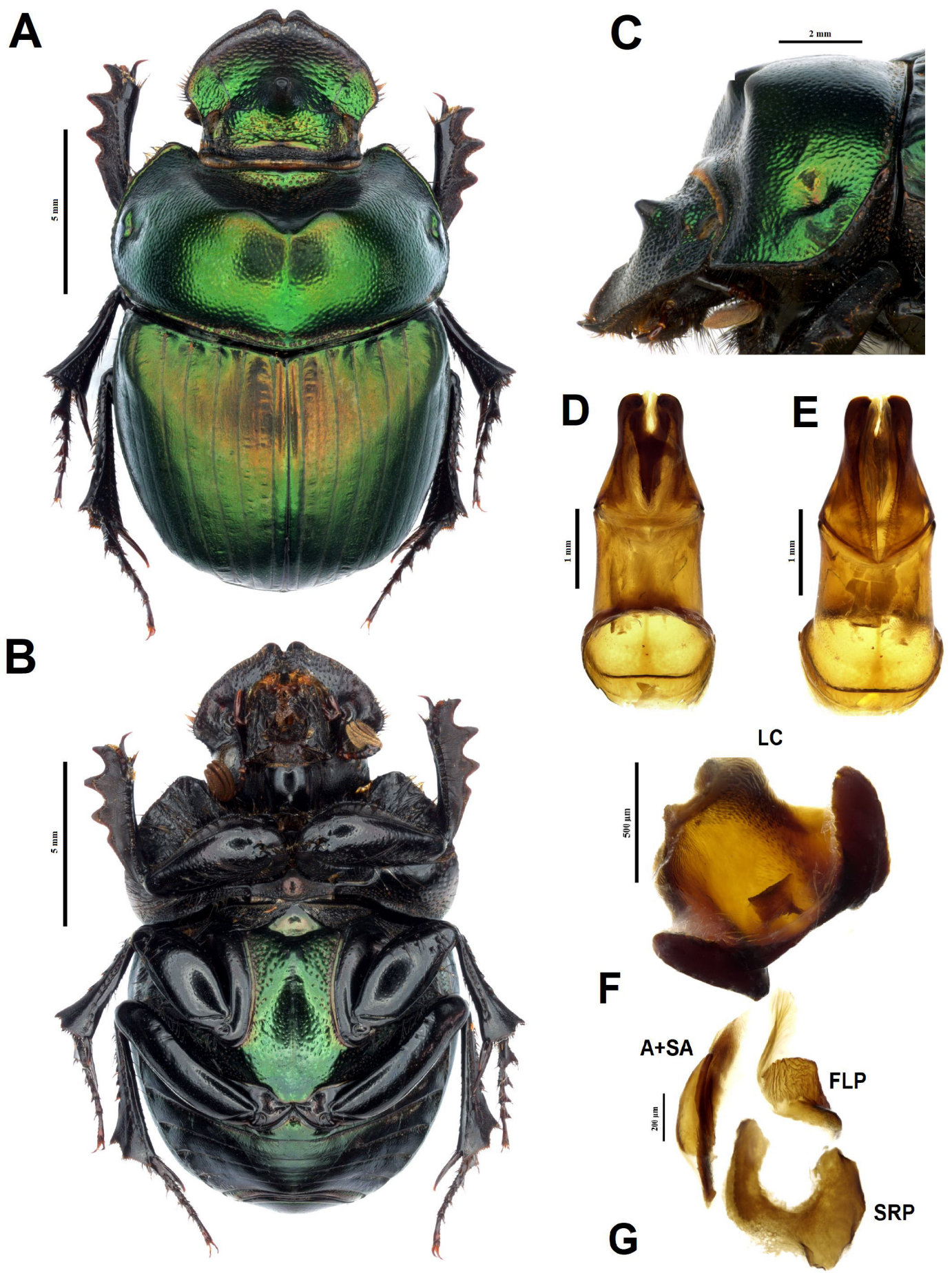

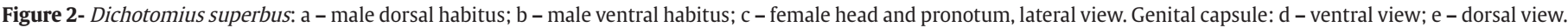

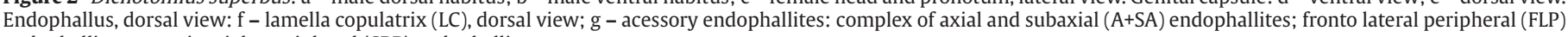
endophallites; superior right peripheral (SRP) endophallites.

a conical process, apically emarginate. Antenna with nine antennomeres, antennal club elongated, covered by dense short pubescence and longer scattered setae. Pronotum. Wider than long. Antero-median portion widely excavated. The surface of pronotal excavation with dense ocellate punctures (Figs. 2a, c). Disc distinctly convex, anterior margin with a pair of knobs separated by a broad central emargination; surface densely coated with shallow punctures (Figs. 2a, c). Margins distinctly marginate; margin of the lateral margin irregularly serrate throughout. Posterior margin with a band of irregularly spaced ocellate punctures. Paramedian fovea delimited on lateral external margin by a small tubercle. Anterior angle rounded. Median longitudinal sulcus moderately impressed on the disc. Hypomeron. Not excavated anteriorly and lacking transverse carina medially. Median portion almost entirely smooth, except by the presence of scattered seitiferous punctures. Anterior portion densely covered by setiferous punctures, except on glabrous area near anterolateral portion of the circumnotal ridge with ocellate punctures spaced by approximately their diameter. Posterior portions densely coated with ocellate punctures, generally bearing short erect setae. Lateral margin with 
a row of deep impressed setiferous punctures, larger and less dense near the lateral margin. Prosternum. Surface with coarse punctures, central portion distinctly convex. Mesoventrite. Smooth medially, lateral portions densely covered by setiferous ocellate punctures. Meso-metaventral suture regularly arched. Mesepimeron and metepisternum. Covered by dense setiferous punctures, setae long. Metaventrite. Surface with green metallic reflections. Anterior margin with a deeply V-shaped impression medially. Anterior lobe with a median angulate process. Metasternal disc feebly punctate, median longitudinal sulcus moderately impressed. Anterior lobe glabrous and with ocellate punctures more concentrated laterally. Lateral lobes covered by dense setiferous punctures, setae long. Elytra. Striae wide, shallowly impressed along their length, not punctate. Interstriae with fine sculpture and inconspicuous punctures densely distributed. Sixth and seventh interstriae strongly elevated on basal onesixth. Third and fourth interstriae widely impressed on basal one-sixth. Eighth stria carinate and punctate, punctures larger and deeper than stria, irregularly spaced throughout the stria. Ninth stria visible only at the apical third. Legs. External margin of protibiae quadridentate. Apical spur of protibiae acute, curved down on apical third. Ventral surface of profemur with ocellate setiferous punctures along the midline. Ventral surface of meso- and metafemurs with sparse shallow punctures. Ventral surface of meso- and metatibiae densely coated with fine punctures on apical half. Meso and metatibia widely expanded apically. Apical margin of metatibia oblique, distinctly angulate medially. Apical spur of metatibiae apically truncate. Abdomen. Ventrites narrowed medially; surface smooth, except by anterior margin with a single row of small ocellate punctures. Fifth ventrite half the length of sixth medially. Pygidium. Slightly convex. Anterior margin with a row of ocellate punctures. Disc covered by sparse shallow punctures. Genital capsule. Paramera symmetric and moderately narrowed forward the apex. Apex of paramera truncate, left paramere lacking projection or hook on external margin. Ventral sclerotized valves of paramera approximately h-shaped. Endophallus: lamella copulatix (LC) approximately double hook-shaped, medianposterior portion with a tongue-like projection one-fifth as long as the LC (Fig. 2e). Superior right peripheral endophallite (SRP) C-shaped (Fig. 2e). Fronto-lateral peripheral endophallite (FLP) comma-shaped with an anterior projection (Fig. 2e). Complex of axial and subaxial ( $A+S A)$ endophallites elongated and curved (Fig. 2e). Female. It differs from male by the head surface with transverse rows of deeply impressed punctures. Anteromedian portion of pronotum with shallower excavation. Sixth abdominal ventrite with half the length of fifth medially.

\section{Discussion}

Luederwaldt (1929) had grouped the large species of $D$.(Selenocopris) that exhibited a single cephalic process, emarginated at the apex, and a pronotum with abundant pilosity on lateral sides as well as a strong anterior declivity into the "Nisus" section (currently D. nisus species group). The author himself pointed out his difficulties to distinguish the "Nisus" section using few characters, given that the species within it exhibit considerable variation in color (black to colored specimens), body lenght (16-26 mm), shape of the clypeal teeth (rounded to acute) and shape of male pronotum (with or without anterior declivity) (Luederwaldt 1929).

Following the currently used classification of $D$.(Selenocopris) species groups (Nunes and Vaz-de-Mello, 2019), the species originally included in the "Nisus" section now belong to the following species-groups: $D$. nisus and D. superbus are isolated species not formally assigned to any species group; $D$. fimbriatus exhibits most characters of the D. crinicollis species group, such as a smooth clypeal margin, male cephalic process produced into a transverse carina and metasternal borders, mesepisternum, and hypomeron with abundant red pilosity;
$D$. lycas fits on the $D$. carbonarius species-group because it exhibits a metallic-copper collored dorsum, chagreened pronotum and elytra, and a head with a single conical process. Within D. (Selenocopris), $D$. nisus and $D$. superbus share a single cephalic process on the male head, coupled with a bilobated pronotum. Our comparative study of male genitalia revealed that despite their external resemblance, the two species have strongly diverged in the morphology of the aedeagus (genital capsule and endophallites). In D. nisus, the aedeagus seems more longitudinally elongated in comparison with $D$. superbus, and the apex of the parameraare strongly asymmetric. Asymmetric paramera have not been described for any other species of the genus Dichotomius.

Regarding the endophallites, the LC of the two species have similar ground-plans, with some remarkable interspecific differences (see redescriptions). In both species, the LC is a sub-rectangular plate with a pair of laminar projections in its inferior margin. Inside the endophallus of $D$. nisus, the LC is positioned longitudinally in relation to the genital capsule and the laminar projections are parallel to the same axis. By contrast, in D. superbus the LC and, consequently, the laminar projections are positioned obliqually to the genital capsule. Within Dichotomius, the LC seems assumes at least three different positions inside the endophallus because, besides the two positions described here, Arias-Buriticá and Vaz-de-Mello (2019) also illustrated the LC of the species in the "buquet" group positioned transversally to the genital capsule.

The LC of both $D$. nisus and $D$. superbus exhibits a median projection in the sub-rectangular plate. In D. superbus, the LC has a short median projection near its inferior border (Fig. 1f), while in D. nisus the same median projection is three times longer (Fig. 1f). As an assessment of homology of endophallites within Dichotomiini has never been performed, alternative interpretations of LC morphology may arise. One might be that the median projection of $D$. nisus is not homologous to that in D. superbus, but rather a remnant part of the main plate of the $\mathrm{LC}$, which in this species is not completely sclerotized at its medial portion.

The LC ground-plan has been useful for diagnosing others species-groups within Dichotomius. Arias-Buriticá and Vaz-de-Mello (2019) redefined the "buqueti" group of $D$. (Dichotomius) based on the presence of a sclerotized plate between paramera and different LC grand-plans found in the species formely included this group. Valois et al. (2018) found the same LC ground-plan in all species of the "sericeus" group of $D$. (Selenocopris). However, future phylogenetic studies need to be conducted to test the phylogenetic value of the endophallites and to provide the necessary assessment of homology between its parts.

In the original description of D. nisus, Olivier (1789) stated "Cayenne" [French Guiane] as type locality. After Olivier's publication, this information was replicated in latter works as catalogues and checklists (Olivier, 1790; Fabricius, 1801; Harold, 1869). New country records to D. nisus from Brazil and Argentina become available only in the Gillet's catalogue of Scarabaeidae in 1911. However, it was in Luederwaldt (1929) that the known distributional range of $D$. nisus was greatly expanded to Paraguay and almost all Brazilian regions, except the midwest. Currently, D. nisusis considered a coprophagous-generalist species (Hernandez, 2007; Vieira et al., 2008; Silva et al., 2014), found in dry and anthropized habitats (Nunes et al., 2012; Filgueiras et al., 2015) from Venezuela to northern Uruguay (Lozano, 2010; Ferrer-Paris et al., 2013). According to our available collection data, this species has become widely spread in natural dry habitats and in places where forests have been converted into pastures or have suffered from intense clearing by human activities. There are records of $D$. nisus in the savannas from Venezuela, Colombia and Roraima state(Brazil)(Fig. 3). These populations seems geographicaly isolated from others by the presence of the surrounding matrix of primary rainforest, which is the main factor limiting the distribution of D. nisus in the north of South America. 


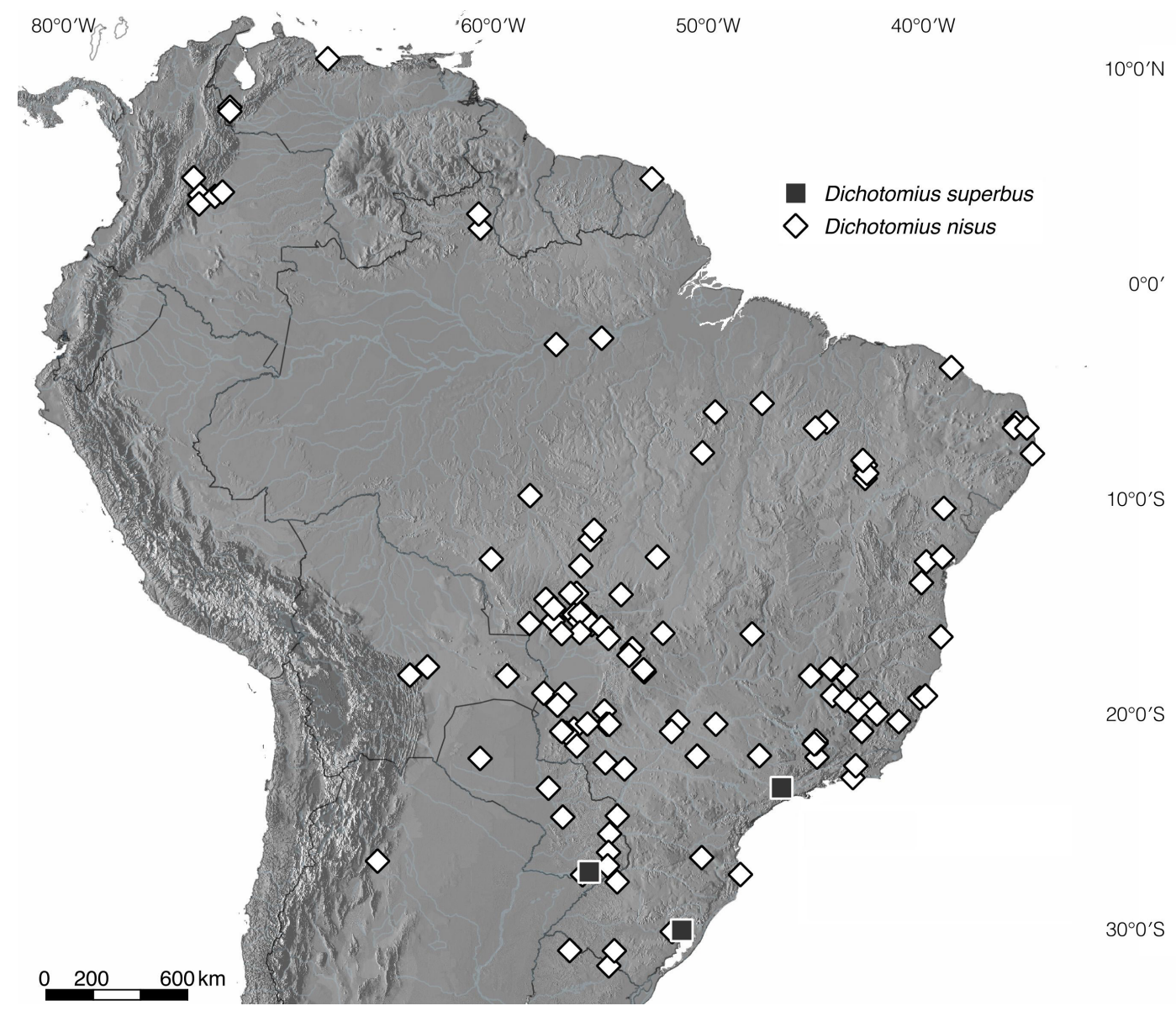

Figure 3- Distribution map of Dichotomius species here studied. Black square, Dichotomius superbus; white lozenge, Dichotomius nisus.

However, no significant differences were found regarding both the external morphology and the comparison of genitalia. The Venezuelan/Colombian group exhibited small differences only regarding the size of the genital capsule, being smaller and more robust than in other populations, but beetles from this groupstill exhibit the left paramere with a strong apical hook and show no difference between the genital capsule and the lamella copulatrix endophallite. This trait is shared with rest of the large group formed by Bolivia, Paraguay, Argentina, Uruguay and the other states from Brazil south of the Amazon River.

One of the main contributors to the degradation of the Amazon rainforest is its deforestation for the establishment of pastures and soybean cultivation (Malhi et al., 2008), with pastures being an environment in which many forest species do not adapt due to the hostility of their conditions (Santos-Filho et al., 2012). The Arc deforestation, as it is known, concentrates most of the deforested areas in the Amazon (INPE, 2005). New conditions originating from human activities may favor some species and affect the total diversity of the landscape (Quintero and Halffter, 2009). This may, in turn, leads to a reduction in species richness, causing a change in the composition of the species (Laurance et al., 2011). Dichotomius nisus occurs in the middle of the state of Amazonas and may have arrived there and in dry areas in French Guiana through corridors (maybe coastal or riverine open-habitat corridors) or (unlikely) as a consequence of deforestation. By contrast, our available collection data regarding $D$. superbus suggest this species has a small distribution range compared to D. nisus, occurring in the Parana forest in Brazil and the chachoan provinces in Argentina (Fig. 3). In two labels of the holotype there is a word, that apparently is "Pará" [probably Pará State in Brazil]. However, Felsche (1901) wrote in the original description of $D$. superbus that the specimen beared no label informing distribution data. Therefore, there is no proof that those labels have not been added to the specimen in later years. Moreover, these labels are likely incorrect, since this species has not been recorded again in the Pará territory or nearby areas.

\section{Acknowledgements}

The following provided fellowships and financial support: Coordenação de Aperfeiçoamento de Pessoal de Nível Superior (CAPES) S.C. (1768693), CNPq for providing research grants for MV (142109/2016-9), FVM is CNPq PQ fellow, and received further support by CNPq (440604/2015-0, 405697/2013-9, 302997/2013-0, 304925/ 2010-1, 306745/2016-0, 484035/2013-4) and FAPEMAT (PRONEM 568005/2014). Examination of European types occurred durying execution of projects granted by CNPq (454152/2011-6 and 202327/2013-2) and by ISYEB, UMR 7205 CNRS/MNHN.

\section{Conflicts of interest}

The authors declare no conflicts of interest.

\section{Compliance with ethical standards}

All fundings are acknowledged and cited above. The authors declare that they have no conflict of interest. This article does not contain any 
studies with human participants or animals performed by any of the authors.

\section{Author contribution statement}

All authors contributed equally to taxonomic revision and decisions in this work. Cassenote and Valois elaborated the plates, list of examined specimens, and redescriptions. Maldaner and Vaz-de-Mello prepared taxonomic history and Maldaner made the distribution map. Cassenote, Valois, and Maldaner wrote the manuscript. Finally, all the authors reviewed and edited the manuscript.

\section{References}

Arias-Buriticá, J. A., Vaz-de-Mello, F. Z., 2019. Redefinition and taxonomic revision of the" buqueti" species-group, Dichotomius Hope, 1838 (Coleoptera: Scarabaeidae: Scarabaeinae). Rev. Bras. Entomol. 63 (1), 43-52. http://dx.doi.org/10.1016/j.rbe.2018.11.002.

Arrow, G. J., 1913. Some new species of Lamellicorn Beetles from Brazil. Ann. Mag. Nat. Hist. 8 (11), 456-466. http://dx.doi. org/10.1080/00222931308693338.

Blackwelder, R. E., 1944. Checklist of the Coleopterous Insects of Mexico, Central America, the West Indies, and South America: Parts 1 and 2. Library of Anthony Marsiglia, Washington.

Burmeister, H., 1846. Genera Quædaminsectorum: Volumen I. Continettabulas XL (Vol. 1). sumtibus A. Burmeister. Berlin.

Fabricius, J. C., 1801. SystemaEleutheratorum: Secundum Ordines, Genera, Species, Adiectissynonymis, Locis, Observationibus, Descriptionibus. Vol. 2. Impensis Bibliopolii Academici Novi, Kiliae.

Felsche, C., 1901. Beschreibungen coprophager Scarabeiden. Dtsch. Entomol. Z. 2, 135-155.

Ferrer-Paris, J. R., Sanchez-Mercado, A., Rodriguez, J. P., 2013. Optimizacióndelmuestreo de invertebrados tropicales: Unejemploconescarabajos coprófagos (Coleoptera: Scarabaeinae) en Venezuela. Rev. Biol. Trop. 61 (1), 89-110. http://dx.doi.org/10.15517/ rbt.v61i1.10941.

Filgueiras, B. K. C., Tabarelli, M., Leal, I. R., Vaz-de-Mello, F. Z., Iannuzzi, L., 2015. Dung beetle persistence in human-modified landscapes: combining indicator species with anthropogenic land use and fragmentation-related effects. Ecol. Indic. 55, 65-73. http://dx.doi. org/10.1016/j.ecolind.2015.02.032.

Gandini, P., Aguilar, C., 2009. Six new species of Dichotomius Hope, 1838 (Coleoptera; Scarabaeidae; Scarabaeinae) from South America and description of the male of Dichotomius camargoi Martínez, 1956. G. Ital. Entomol. 12 (55), 135-164.

Gillet, J. J. E., 1911. Scarabaeidae: Coprinae I. In: Junk, W., \& Schenkling, S. (Eds.), Coleopterorum Catalogus. W Junk, Berlin, Germany, pp. 1-100.

Harold, E., 1869. Révision des espèces qui rentrent dans le genre Pinotus Erichs. Abeille. 6, 123-144.

Hernandez, M. I. M., 2007. Besouros Escarabeíneos (Coleoptera: Scarabaeidae)da Caatinga Paraibana, Brasil. Oecol. Bras. 11 (3), 356-364. http://dx.doi.org/10.4257/oeco.2007.1103.06.

Instituto Nacional de Pesquisas Espaciais - INPE, 2005. Monitoramento da Floresta Amazônica Brasileira por Satélite: Projeto PRODES. INPE, São José dos Campos. Available in: www.dpi.inpe.br/prodesdigital (accessed 20 July 2019).

International Commission on Zoological Nomenclature, 1999. International code of zoological nomenclature. 4th ed. (adopted by the International Union of Biological Sciences). La Garangola, Padua, Italy: 306 pp.

Laurance, W. F., Camargo, J. L. C., Luizão, R. C. C., Laurance, S. G., Pimm, S. L., Bruna, E. M., Stouffer, P. C., Bruce Williamson, G., Benítez-Malvido,
J., Vasconcelos, H. L., Van Houtan, K. S., Zartman, C. E., Boyle, S. A., Didham, R. K., Andrade, A., Lovejoy, T. E., 2011. The fate of Amazonian forest fragments: a 32-year investigation. Biol. Conserv. 144 (1), 56-67. http://dx.doi.org/10.1016/j.biocon.2010.09.021.

Lozano, C., 2010. Nuevos registros de escarabajos coprófagos para laOrchila, Sierra de Perijá, Venezuela. Boletín Cent. Investig. Biologicas. 44, 83-89.

Luederwaldt, H., 1914. Biologia de várias espécies de Pinotus de São Paulo. Rev. Mus. Paul. 9, 365-370.

Luederwaldt, H., 1922. Neue brasilisanische Pinotus-Arten. Rev. Mus. Paul. 1, 9.

Luederwaldt, H., 1929. As espécies brasileiras do gênero Pinotus. Rev. Mus. Paul. 16, 603-776.

Luederwaldt, H., 1931. Novos subsídios para o conhecimento do gênero Pinotus. Rev. Etol. 1, 297-304.

Malhi, Y., Roberts, T., Betts, R. A., Killeen, T. J., Li, W., Nobre, C., 2008. Climate change, deforestation, and the fate of the Amazon. Science 319 (5860), 169-172. http://dx.doi.org/10.1126/science.1146961.

Martínez, A., 1951. Notas Coleopterologicas V. An. Soc. Cient. Argent. 92, 138-142.

Nunes, R. V., Vaz-de-Mello, F. Z., 2019. Taxonomic revision ofDichotomius(Cephagonus)Luederwaldt 1929 and the taxonomic status of remaining DichotomiusHope 1838 subgenera (Coleoptera: Scarabaeidae: Scarabaeinae: Dichotomiini). J. Nat. Hist. 53 (37-38), 37-38, 2231-2351. http://dx.doi.org/10.1080/00222933.2019.1692088.

Nunes, R. V., Vaz-de-Mello, F. Z., 2013. New brachypterous species of Dichotomius Hope, with taxonomic notes in the subgenus Luederwaldtinia Martínez (Coleoptera: Scarabaeidae: Scarabaeinae). Zootaxa 3609 (4), 411-420. http://dx.doi.org/10.11646/zootaxa.3609.4.3.

Nunes, R. V., Frizzas, M. R., Vaz-de-Mello, F. Z., 2012. Scarabaeinae (Coleoptera: Scarabaeidae) of a rupestrian field at Caatinga, Distrito Federal, Brazil: commented list of species. Biota Neotrop. 12 (4), 125-129. http://dx.doi.org/10.1590/S1676-06032012000400013.

Olivier, G. A., 1789. Entomologie, Ou, Histoire Naturelle des Insectes: Avec Leurs Caracteres Generiques et Specifiques, Leur Decription, Leur Synonymie, et Leur Figure Enluminee. Baudoin, Paris.

Olivier, G. A., 1790. Encyclopedie Methodique, Ou, Par Ordre De Matiéres; Par Une Societé De Gens de Lettres, De Savans, Et D’Artistes. Panckoucke Libraire, Paris.

Quintero, I., Halffter, G., 2009. Temporal changes in a community of dung beetles (Insecta: Coleoptera: Scarabaeinae) resulting from the modification and fragmentation of tropical rain forest. Acta Zool. Mex. 25, 625-649.

Santos-Filho, M., Peres, C. A., Silva, D. J., Sanaiotti, T. M., 2012. Habitat patch and matrix effects on small-mammal persistence in Amazonian forest fragments. BiodiversityandConservation. 21 (4), 1127-1147. http://dx.doi.org/10.1007/s10531-012-0248-8.

Silva, R. J., Coletti, F., Costa, D. A., Vaz-de-Mello, F. Z., 2014. Rola-bostas (Coleoptera: Scarabaeidae: Scarabaeinae) de florestas e pastagens no sudoeste da Amazônia brasileira: levantamento de espécies e guildas alimentares. Acta Amazon. 44 (3), 345-352. http://dx.doi. org/10.1590/1809-4392201304472.

Tissiani, A. S. O., Vaz-de-Mello, F. Z., Campelo-Júnior, J. H., 2017. Besouros rola-bostas das pastagens brasileiras e chave para identificação dos gêneros (Coleoptera: scarabaeidae). Pesqui. Agropecu. Bras. 52 (6), 401-416. http://dx.doi.org/10.1590/s0100-204x2017000600004.

Valois, M. C., Harada, L., Vaz-de-Mello, F., Silva, F., 2018. Synopsis of the genus DiabroctisGistel, 1857 (Coleoptera: Scarabaeidae: Scarabaeinae) with a new species description. Insect Syst. Evol. 1, 1-28. http:// dx.doi.org/10.1163/1876312X-00002203.

Vaz-de-Mello, F. Z., 2000. Estado actual de conhecimento dos Scarabaeidae s. str.(Coleoptera: Scarabaeoidea) do Brasil. In: Martín 
Piera, F., Morrone, J.J., Melic, A. (Eds.), Proyectolberoamericano de Biogeografía y Entomología Sistemática: PRIBES 2000: Trabajos del 1er Taller Iberoamericano de Entomología Sistemática. Sociedad Entomológica Aragonesa, España, pp. 183-195.

Vaz-de-Mello, F. Z., Edmonds, W. D., Ocampo, F. C., Schoolmeesters, P., 2011. A multilingual key to the genera and subgenera of the subfamily
Scarabaeinae of the New World (Coleoptera: scarabaeidae). Zootaxa 2854 (1), 1-73. http://dx.doi.org/10.11646/zootaxa.2854.1.1.

Vieira, L., Louzada, J. N. C., Spector, S., 2008. Effects of degradation and replacement of Southern Brazilian Coastal Sandy Vegetation on the dung beetles (Coleoptera: scarabaeidae). Biotropica 40 (6), 719-727. http://dx.doi.org/10.1111/j.1744-7429.2008.00432.x. 